\title{
Understanding Family Connections and Help Seeking Behavior in Chinese American Immigrant Adults who Attempt Suicide
}

\author{
Rose Wong ${ }^{*}, 1$, Dong Li Hou ${ }^{2}$ and Evaon Wong-Kim ${ }^{1}$ \\ ${ }^{I}$ Department of Social Work, California State University, East Bay, USA \\ ${ }^{2}$ Kaiser Permanente Union City, USA
}

\begin{abstract}
Aim: This study explores the influence of beliefs, values and norms in Chinese family culture as they relate to attitudes and beliefs about mental health and mental health services. It examines family and acculturative stressors occurring in the immigrant's social context on attitudes about suicidal distress and help-seeking behaviors, and focuses on appropriate forms of services for suicidal behaviors among adult immigrants experiencing suicidal behaviors.

Methods: The study design is descriptive, using a qualitative approach. Six Chinese American immigrant adults who had attempted suicide participated in semi-structured interviews in Mandarin or Cantonese. The content was analyzed using a constant comparative approach.

Results: Study participants exhibited various reactions to suicidal distress, attitudes about needing help and usefulness and relevance of existing intervention strategies and services that reflected influences of Chinese family culture. Interactions with family members negatively impacted study participants' attitudes about using services and hindered pathways to care. Acculturative stressors, along with weak family ties and the absence of strong community networks exacerbated the strategies for effective services.

Conclusion: This study raises the question of the efficacy of several Western-culture based service delivery models on help-seeking behaviors on such populations. Furthermore, the study discusses ethnic sensitive approaches with core roles for family, peers and community for supporting those at risk of attempting suicide and linking them to appropriate community-based services.
\end{abstract}

Keywords: Culture, Chinese, family, help-seeking behavior, immigrants, suicide, qualitative, mental health.

\section{INTRODUCTION}

Family structure, dynamics and the family's response to the dominant culture, including the rate of family acculturation, is central to providing quality ethnic sensitive community mental health services. This is the case especially for those exhibiting suicidal behaviors. In the Chinese American population, lifetime rates of suicide attempts and suicidal ideation are, respectively, $3.2 \%$ and $10.6 \%$ [1], and the suicide rate among all Asian Americans is 6.5 per 100,000 [2]. Although these rates are lower than national estimates for the general population, respectively $4.6 \%, 13.5 \%$ [3] and 11.5 per 100,000 [4], suicide was the eighth leading cause of death for Asian and Pacific Islanders, but the tenth leading cause of death for Whites, accounting respectively for $2.0 \%$ and $1.5 \%$ of total deaths [4]. Older adult women, ages 65-84 years, furthermore have the highest rate of suicide (6.5 per 100,000) compared with all racial and ethnic groups [5], and older adults overall report very high rates of suicidal ideation and attempts in primary care and community studies [6].

*Address correspondence to this author at the Department of Social Work, California State University, Hayward, 25800 Carlos Bee Boulevard, Hayward, CA 94542; Tel: (510) 885-4916; Fax: (510) 885-7580;

E-mail: rose.wong@csueastbay.edu
Research on Asian Americans, especially Asian immigrants is woefully inadequate. What little research that is available suggests that Asian Americans are not obtaining timely and appropriate mental health care. This population has the lowest rates of mental health service use compared with all other racial and ethnic populations [7, 8]. As an example, $68.7 \%$ of Asian Americans with past-year depressive disorder did not access mental health treatment compared with $40.2 \%$ of White Americans [9]. Chu, Hsieh and Tokars [10] found that Asian Americans with suicidal ideation or suicide attempts exhibited a low likelihood of contacting mental health professionals other than medical professionals. Also, $35.7 \%$ of suicide attempters reported that they never sought professional care [10].

The limited research that is available (much of which we review in this paper) suggests that culture, levels of acculturation and other acculturation-related factors influence Asian, particularly Chinese family attitudes about suicidal distress and help-seeking attitudes. Values related to "face" or shaming the family, tradition, cultural norms and weakening of social support networks, all interact to influence Chinese American immigrants' awareness and willingness to admit suicidal distress $[11,12]$. Consequently, these variables all influence the willingness and decisionmaking attitudes of Chinese Americans to obtain needed services [10-15]. This problem of poor linkage to care is part 
of a broader problem of underutilization of mental health services among Asian Americans [10].

In this study, we build on the importance of cultural understanding to generate knowledge that can support improving linkage to care and culturally sensitive forms of services for Chinese American immigrants with suicidal behaviors. Through qualitative interviews with six adults who attempted suicide, we explore the impact of cultural influences on attitudes about suicide and help-seeking behaviors. Particularly, we focus on those attitudes and behaviors related to family dynamics, the social context of acculturation, adaptation to a new society and trust and utilization of community based mental health services. We also examine the influence of culture on reasons for attempting suicide as background knowledge and to assist in understanding help seeking and attitudes about services.

\section{CULTURAL FACTORS RELATED TO FAMILY AND COMMUNITY CONTEXTS}

A review of literature suggests that the Chinese emphasis on family cohesion plays an important role in the manifestation of suicidal behaviors and help seeking. Family cohesion is characterized by the emphasis on fulfilling familial obligations, subordinating one's needs to those of the family, and maintaining familial loyalty [16]. Because of the importance of family interdependence and respect for elders in Chinese families, conflict within one's family is an influential factor in suicidal behavior [15, 17, 18]. Problems in family relationships, particularly not adequately fulfilling family roles and responsibilities and "not fitting in" represent a major threat to one's sense of self and wellbeing [19]. Conflict in the family, bringing negative image to the family or perceived rebelliousness are some of the issues that may trigger psychological distress and suicidal expression. Often in the family setting these triggers and the severity of the stress they cause go unrecognized $[11,15,20]$.

Family factors have also been shown to impact help seeking. Several studies found that Chinese immigrants who attempted suicide did not pursue supportive services because of feelings of shame for not fulfilling family expectations or role obligations and fear of judgment by family and peers $[11,14]$. Another explanation of the reluctance to seek help is the view of mental illness and suicide as 'character weakness' where communicating one's personal problems may be experienced as disgracing one's family and fearing judgment of personal failure [21, 22].

The literature also highlights how the community context may influence suicidal and help seeking behaviors. For example, fear of what neighbors will think and attitudes about exposing personal or family problems to strangers all have to be viewed within a cultural context, i.e., how are such issues seen through the cultural lens of the Chinese family $[15,20]$. Equally important, how do such family factors intertwine with community factors? For example, in the case of immigrants, there may be community support available, but access to that support and its quality may be dependent on the immigrant's level of acculturation and ability to integrate and make use of those resources. Problems with English proficiency, lack of transportation, or mistrust of dominant culture community institutions are often found to exacerbate family difficulties and help seeking patterns among Chinese immigrants with suicidal behaviors $[11,12,15]$. Chung [11] observed that immigrants who attempted suicide were individuals who were socially isolated and marginalized with a weak support network of families, friends and community institutions. These immigrants not only lacked knowledge about community resources, but their difficulties with the English language also exacerbated family conflict. Add to these dynamics the possibility of intergenerational conflict with their children and the stress in compounded $[11,15]$.

From the perspective of the Chinese family and culture, together with community and acculturation-related factors, it appears as if a person in need of mental health services is caught in a double bind; on the one hand he or she is not aware of the need for such intervention and, on the other hand, seeking such intervention requires the approval and support of the family and integration into the community $[20,23]$. However, the need for such care goes mainly unrecognized or unappreciated in both the individual and the family. Chung's study $[11,12]$, examining the role of family and community in linkage to care among Chinese immigrants with suicidal behaviors, found that when immigrants disclosed suicidal ideation, family members did not direct them to seek help, and primary care physicians also failed to provide them with psychiatric referrals.

\section{CULTURAL FACTORS THAT HINDER AWARENESS AND EXPRESSION OF THE NEED FOR HELP}

Chinese Americans with suicidal ideation expressed little awareness of the need for help regarding their emotional or mental distress $[10,14,15]$. In this regard their selfperception of the need for mental health treatment was no different from those expressed by individuals who had a mental disorder without a history of suicide [10]. This apparent lack of recognition of the need for treatment when experiencing emotional distress could be attributed to a number of factors. For example, it could be attributed to the Chinese immigrants' interpretation of their symptoms as not being worthy of professional attention. It could be related to the stigma of mental illness in the Chinese community or shame of "the problem" being revealed publicly. Or, it could be manifested as somatic in nature [24, 25]. These interpretations lead the immigrant to deny the need for care until the condition has deteriorated or the immigrant might not recognize the real nature of the problem and seek help in primary care and community settings rather than specialty mental health services $[14,17,20,26]$. Further blockage to obtaining care occurs when such settings tend to not provide mental health care and the immigrant must face a complicated referral system. Lin and Cheung [27] observed that Chinese immigrants were only able to make the connection between mental illness, suicide attempts and the need for help while in treatment after suicide attempts.

Traditional Chinese culture and tradition may also create a barrier to mental health services. A few studies have observed the influence of the Chinese emphasis on emotional restraint on the expression of psychological distress and help seeking. In Confucian culture, restraint of emotional expression serves the purpose of attaining individual and communal (or family) harmony, and the private experience 
of harmony through emotional regulation contributes to the establishment of social harmony [28]. With regard to suicidal behavior, Asian Americans are referred to as "hidden ideators"; they are less likely to seek professional help for psychological distress and to disclose suicidal ideation without a clinician's probing [13]. The Chinese value placed on emotional restraint is a cultural explanation for such behaviors of hiding suicidal ideation [29]. Hidden ideation was common in a qualitative study of suicidal behaviors among community-residing older adult Chinese American immigrants with chronic illnesses [14].

\section{WESTERN-BASED MODELS OF MENTAL HEALTH HELP-SEEKING}

Three dynamic theoretical models on help seeking behaviors in the general population guide this study's exploration of the influences of Chinese culture on immigrants' help seeking for suicidal distress. Dynamic models are of interest to exploring finer-grained cultural influences because they focus on the process of help seeking as opposed to static, deterministic models that explain barriers to care by focusing on when and how people use the formal system of care (i.e., specialized mental health and general medical care) and on profiles of service users with the assumption of rational decision-making processes [30].

The first model, the cycle of avoidance model, highlights non-help-seeking, particularly forms of ambivalence and difficulties experienced during the pathway to care by people experiencing mental distress [31]. Central to this model, which takes an interpretive approach, are social meanings attached to help seeking and treatment, the purposeful action of individuals, and lay conceptions of mental distress. Although the model is developed among young male and female adults (ages 16-24 years) with mental distress, its authors assert that the model could serve as a theoretical template for explaining non-help-seeking in other age groups and populations.

Secondly, the Network-Episode Model focuses on the nature of the process and timing by which individuals seek treatment in the mental health system. According to this model, help seeking is a social process managed by the individual's social support networks in the community, the treatment system, and social services agencies [30]. How people respond to illness is a process of social influence and a result of individual action. Individuals are viewed as users of common-sense knowledge and culturally based routines, and they seek out and respond to others when they come to experience unusual or psychiatric symptoms. Whether someone eventually receives formal care depends not only on the treatment system, but also the social support system, comprised of the lay system of care (friends, family, and coworkers), the folk system of religious counselors and alternative healers, and the social services system (police, teachers, clergy, and support groups). According to this model, one's patterns of care over time (called an 'illness career'), is affected by the person's social support system. If the network generally has a positive attitude toward mental health service use, the individual is more likely to use services. On the other hand, the network can also deter service use or have no effect at all. This model, conceptualized based on diverse empirical findings, is designed for all age and gender groups and racial and ethnic populations as it takes into account each specific group's beliefs about the form of health care [32, 33].

Third, the concept of help-seeking pathways by Rogler and Cortes [34] focuses directly on the role of culture in help seeking. Considering distress as a culturally shaped phenomenon, the application of this concept is viewed as useful for gaining knowledge to make mental health care accessible among underserved populations. A pathway is the distressed individual's sequence of contacts with people and organizations that result from his or her own efforts and those of significant others to seek help and from the response provided to such efforts. The pathway, comprising these interpersonal help-seeking and help-receiving efforts, includes cultural interpretations of the evolving distress, attempted therapeutic and social interventions by laypersons and professionals, and referrals to other help-giving groups. With the onset of distress commencing the pathway, the direction of the pathway is the ordering of individuals and organizations contacted, and its duration is the time lapse from the time of initiating help seeking to the formation of contacts. From the onset, psychosocial and cultural factors impinge upon the severity and type of mental health problem, and these factors continue interactively to shape the pathway's direction and duration. According to this concept, a person monitors his or her own psychological changes, interprets the changes, takes actions, and then re-evaluates the symptoms after interaction with experts, with the subjective experience and meanings attributed to the experience viewed as imperfectly related to the clinical facts of the illness. The concept of help-seeking pathways was developed to integrate knowledge about the use of mental health care, with the aim of making mental health care more accessible among underserved populations, and is applicable to all social, cultural, age and gender groups.

Although no specific hypotheses are drawn based on the three models reviewed in this exploratory study, the models are all expected to be viable for clarifying the influence of factors related to culture, family and acculturation on immigrants' pathways to care. The cycle of avoidance model is expected to be useful for highlighting how social and cultural meanings can contribute to creating difficulties which lead to non-help-seeking, especially considering the family's denial of the need for care and basic cultural factors that hinder help-seeking reported in the literature. Considering that family and acculturative context have been identified as key factors that hinder help seeking and the strong avoidance or rejection of existing mental health services suggested by low rates of service use among Chinese immigrants, the Network-Episode Model may be useful for examining the cultural processes and routines occurring at the intersection of family, community, treatment system and social services, particularly how these contexts interplay and contribute to the overall problem. Finally, Rogler and Cortes' culturally focused concept of helpseeking pathways may be helpful for examining the diverse points on the care pathway where cultural interpretations influence the timing of a Chinese immigrant's expressions of distress and help seeking actions. 


\section{MATERIALS AND METHODOLOGY}

\section{Sampling and Recruitment}

Six study participants were recruited within one week after their suicide attempts. All study participants met the following criteria: born outside the United States; Chinese as first language; fluency in Mandarin or Cantonese; at least 18 years of age at time of suicide attempt; made a suicide attempt that required emergency medical intervention; and resident of the San Francisco Bay Area. Three participants were recruited from a hospital emergency room where they were evaluated after a suicide attempt. Two participants were recruited through the investigators' professional contacts. One was recruited through a social service agency where the participant received services.

\section{Data Collection}

The second author conducted semi-structured interviews at the hospital or in participants' homes after obtaining written consent. Five interviews were in Mandarin and one in Cantonese. The interviews began with background questions on personal and family psychiatric history, including history of suicidal behaviors, general medical conditions and current medications, alcohol and substance abuse history, and current suicidal ideation and plans. The semi-structured interview guide covered the following topics: reasons for the recent suicide attempt and means of survival; availability and role of social support networks in obtaining care; family and friends' reactions to suicidal expressions and suicide attempts; services received before and after suicide attempts; ideas on helpful resources to prevent future attempts; and current life stresses and coping approaches. The Institutional Review Board of California State University, East Bay approved the study protocol.

\section{Data Analysis}

Interviews were audio-recorded and transcribed in Chinese. Because the second and third authors are fluent in Cantonese and Mandarin, interviews conducted in either of these major dialects of Chinese language were transcribed in Chinese to preserve meaning and to enhance the trustworthiness of the data. The Chinese transcripts were then translated into English by the second author to facilitate coding. Narratives in English were entered into ATLAS.ti qualitative analysis software and analyzed using a constant comparative approach [35]; this approach was used to identify concepts, develop categories, and pinpoint themes that provide a structured framework for organizing the data. In particular, this analytic process involved open coding (i.e., identifying a list of categories from a line-by-line analysis of the transcripts) and axial coding (i.e., collapsing the categories generated through open coding into unifying themes).

\section{Sample Characteristics}

Four women and two men comprised the sample. The Table 1 summarizes characteristics of study participants. Time of residence in the U.S. ranged from 10 to 21 years with a mean of 18.2 years. Among all the participants, only Mr. Zhen received social services.

Table 1. Characteristics of study participants.

\begin{tabular}{|c|c|c|c|c|c|c|}
\hline Pseudonym & Mr. Li & Mr. Zhen & Mrs. Wu & Mrs. Wong & Mrs. Chen & Mrs. Lam \\
\hline Gender & Male & Male & Female & Female & Female & Female \\
\hline Age & 84 & 73 & 51 & 47 & 44 & 34 \\
\hline Country of origin & Taiwan & China & Taiwan & China & Taiwan & Malaysia \\
\hline $\begin{array}{c}\text { Age at } \\
\text { immigration }\end{array}$ & 74 & 55 & 30 & 27 & 20 & 18 \\
\hline Languages & Mandarin Chinese & Mandarin Chinese & Mandarin Chinese & Cantonese Chinese & $\begin{array}{l}\text { Mandarin Chinese } \\
\text { and English }\end{array}$ & $\begin{array}{c}\text { Mandarin Chinese } \\
\text { and English }\end{array}$ \\
\hline Education & College & College & Unknown & High school & Some college & College \\
\hline Marital status & Married & Widowed & Married & Separated & Separated & Married \\
\hline $\begin{array}{l}\text { Living } \\
\text { arrangement }\end{array}$ & $\begin{array}{c}\text { With wife in senior } \\
\text { housing }\end{array}$ & $\begin{array}{c}\text { Alone in board and } \\
\text { care home }\end{array}$ & $\begin{array}{l}\text { With husband and } \\
\text { children in own } \\
\text { home }\end{array}$ & $\begin{array}{l}\text { With children in } \\
\text { own home }\end{array}$ & $\begin{array}{l}\text { With children in } \\
\text { own home }\end{array}$ & $\begin{array}{l}\text { With husband and } \\
\text { children in own } \\
\text { home }\end{array}$ \\
\hline Income & $<\$ 10,000$ & $<\$ 10,000$ & Declined to answer & $\$ 20,000-\$ 39,999$ & $\$ 40,000-\$ 59,999$ & $>\$ 100,000$ \\
\hline Employment & Retired & Retired & House-wife & Retired (early) & $\begin{array}{c}\text { Small business } \\
\text { owner }\end{array}$ & White collar \\
\hline Religion & Christian & Christian & None & Christian & Declined to state & None \\
\hline Suicide attempts & Overdose (twice) & Overdose (twice) & Kitchen knife & Overdose & $\begin{array}{l}\text { Overdose (twice), } \\
\text { smashed car }\end{array}$ & Overdose \\
\hline $\begin{array}{c}\text { Reasons for } \\
\text { suicide attempt(s) }\end{array}$ & $\begin{array}{c}\text { Physical illness, } \\
\text { long-term } \\
\text { depression }\end{array}$ & $\begin{array}{l}\text { Loneliness, } \\
\text { isolation }\end{array}$ & $\begin{array}{l}\text { Children, physical } \\
\text { illness, long-term } \\
\text { depression }\end{array}$ & Physical illness & Marriage & $\begin{array}{l}\text { Marriage, long- } \\
\text { term depression }\end{array}$ \\
\hline
\end{tabular}




\section{RESULTS}

Three of the respondents had made one suicide attempt and three had made two suicide attempts (see Table 1). Only Mr. Zhen experienced suicidal ideation at the time of the interview. Only Mrs. Chen reported alcohol abuse. None of the study participants had mental illness diagnoses before their suicide attempts. Mr. Li, Mrs. Wu and Mrs. Lam were given diagnoses of depressive, bipolar and anxiety disorders after their suicide attempts. Suicide methods included overdose (5 participants) and self-harm with a kitchen knife (1 participant). Mrs. Chen, who attempted suicide by overdosing, made a second attempt by crashing her car into a tree.

\section{Reasons For Attempting Suicide Emphasizing Family Difficulties}

Study participants attributed their suicide attempts to biopsycho-social factors. However, the central theme for every participant was a family-related difficulty, whether or not it was alone or existed together with a physical, health or emotional condition. The main reasons given by study participants for their suicide attempts were chronic health problems and the resulting family burden these problems caused. The problems were as follows: health problems (Mr. Li and Mrs. Wong), marital difficulties (Mrs. Chen and Mrs. Lam), conflict with children (Mrs. Wu), and isolation from family and friends, loneliness and sleep disturbance $(\mathrm{Mr}$. Zhen).

Chronic Illness and Family Burden. Mr. Li, Mrs. Wu and Mrs. Wong cited painful, chronic illness as their reasons for attempting suicide. They reported having exhausted medical options and felt little hope of improving their health in the near future. Mrs. Wong explained that she no longer wanted to live due to cancer and chemotherapy. "It was too hard to go through the ninth chemotherapy treatment. I could not bear it anymore. I really wanted [to die]." Mr. Li explained his unbearable abdominal pain. "I was in such pain that I could not stand it. I had so much pain that I could not fall asleep for two nights. I hated that I was in so much pain that I took the pills to end it all.'

Chronic debilitating illness can create a sense of burden for caretakers in all populations. However, it appears that participants in this study had heightened sensitivities to their burdensome impact on their families. Not wanting to be a burden on family appears to be central to their reasoning and, as the literature suggests, reflects the Chinese cultural value of interdependence. Participants focused strongly on family members' stress and burden, explaining that this also heightened their own stress and depression. Mrs. Wong described how her cancer diagnosis destroyed her family.

"The whole family was broken... and facing the disease. [I felt that] I was a burden to my family because that they had to take care of me. I very much wanted to kill myself so that I wouldn't have to suffer so much and my daughter would not have to work so hard [to take care of me]."

\section{Lack of Awareness of Mental Health Condition Related to Family Denial}

Study participants and their family members persistently were in denial in terms of participants' poor state of mental health. Participants' explanations about how such denial manifested were consistently centered on interrelationships with family members, such as the impact of one's mental state on family members or the role of family members in one's help seeking. Study participants highlighted the central role of the Chinese family in the process of denial and its consequences for accessing professional care.

Both male and female participants failed to acknowledge the existence or severity of their poor state of mental health and its relationship to risks of suicide even when mental illness had long affected their family relationships, as in the cases of Mrs. Wu and Mrs. Lam, who were married and living with their husbands and children, and Mr. Zhen, who was widowed. All these three study participants identified long-term depression as the main reason for their suicide attempts. None, however, were aware of having depression or other mental health problem and none reported that they had sought any professional services prior to their suicide attempts. These three participants were given diagnoses of depressive, anxiety and bipolar disorders only after their suicide attempts. They described experiences of persistent and strong depressive symptoms, especially feelings of sadness and hopelessness in their daily lives. These symptoms were known to their families before attempting suicide but had not been treated. Mr. Zhen described his desperation: "I could not find anything that I could grasp and say, 'That is okay. There is hope'. There was completely no hope. You cannot even think about finding hope; you cannot create it or grasp it. So, I reached that next stage [of suicide]."

None of the interviewee's family members recognized or took study participants' suicidal ideation seriously prior to suicide attempts. Mr. Li was told by a family member that he was looking for attention. Family members thus did not provide help and may have hindered participants' own acknowledgement of needing help. Interviewees were aware that their lives had not been happy but interpreted this as how their lives were supposed to be. Mrs. Chen reflected back upon her heightened emotional state. "At that time I was unhappy, then I was upset [that I had not gotten any acknowledgment and help from my family]." She first attempted suicide in Taiwan when she was 15 years old. Her family took no steps to help her before or after that first attempt.

"I had a very bad relationship with my Mom... I was very seriously thinking about suicide. I went to the rooftop. The building was high. Although it was [only] the fourth floor, it was high. I could only remember the thoughts I had at that time, which was, 'Jump, you will be okay. Jump, you will be okay."

Family's denial of poor mental health was also manifested in study participants' unwillingness to share their family members' mental health issues. This was so for both male and female participants. Only Mrs. Lam shared that her family members had a mental health history while other 
participants denied it without reflection. Mrs. Lam stated that no family members had been diagnosed due to not acknowledging their psychiatric problems. She said, "My mom, my uncle, my grandma, all have it. Very serious, especially my mom and my grandma, but they all think that they don't have any problems, and they refuse to look for help."

\section{Social Context of Family and Community Not Providing Needed Support}

Participants reported difficulties associated with social isolation and adapting to life in the U.S. While acknowledging the economic advantages of living in the U.S., study participants emphasized feelings of strong isolation and distress. Repeatedly, this isolation and distress resulted from loss of family support, poor integration and low self-esteem. This, in part, derived from language difficulties, and conflicts with spouses and children but it was also due to the stresses of acculturation particularly related to possessing a low level of English proficiency.

Mr. Zhen, Mrs. Wong and Mrs. Chen attributed their suicide attempts to a process of weakening of family support and subsequent isolation. Immediately after immigration, they experienced support from parents and siblings who remained in their home countries. As time passed, however, these important connections weakened with less and less contact, and participants became unable to maintain a sense of connection. They suffered strongly, citing the loss of both emotional and physical support. Mrs. Chen said, "I have to do everything for myself in America. It is too hard." Mrs. Wong described her isolation.

"At least it was very convenient [in my home country]. Communication was better and language was convenient, and friends, I could chat with them. If I was not happy, I could vent to them. I have no one to vent to here."

Participants' isolation also increased as they became more overwhelmed with emotional distress because this led to cutting off contact further, leading to more isolation. Mrs. Chen, when feeling lonely and isolated, did not share these problems with her parents and other family members in China, increasing her isolation: "I talked relatively less with them because I did not want them to be worried. Because they were far away, I [even] told them, 'You cannot just fly here [to be with me]'." Mrs. Chen's proficiency in English also failed to assist her with creating a social support network in the U.S. Mr. Zhen explained that his siblings in China had been of great support for him in the past, but they did not call him after his migration. He had to call them if he wanted to maintain contact. Since he became depressed, he stopped contacting anyone, which resulting in no family support at all. Being widowed, furthermore, contributed to his sense of isolation and loneliness.

\section{Attitudes about Use of Medications Dependent on Family and Service Providers}

Mr. Zhen, Mrs. Wu and Mrs. Lam were taking medications after their suicide attempts. Family members and service providers influenced whether their attitudes were positive or negative. Mrs. Wu and Mrs. Lam felt that medication helped them to function and stay calm and made their lives better. They were not suicidal at the time of their participation because of the medication. As their symptoms improved, their relationships with family members improved and they became happier. Mrs. Wu stated that "the medication helps because, at least when I wake up I can say, okay, another day. In the past it was, every day is it." Mrs. Lam described how she had benefited in her social behavior from medication: "After I took this medicine for depression, plus the sleeping pills, it seems that I could sleep better, fewer headaches. I used to have lots of headaches. I would have a bad temper in the morning if I couldn't sleep well. Now, my bad temper was gone."

When Mrs. Wu was initially prescribed medication, she was afraid of taking it because she didn't want to label herself as a "crazy" person. Her husband also called her "crazy" and it made her even more resistant to trying medication. Her second doctor helped her to overcome her fear: "Actually very early on I went to see another doctor, he prescribed anti-depressants for me. I was unwilling to take them for a long time... [my second doctor] said, you know, [mental illness] is no different than a heart attack, or diabetic. It is the same." The doctor's understanding of her experience of mental illness alleviated the stigma of mental illness, allowing her to accept the medications.

Side effects of medications were another concern. Mr. Zhen complained that his medication made him dizzy all day long and he could not walk outside of his residence without supervision from the board and care home staff. Mrs. Wu was bothered by her medication a lot. She stated that after taking the anti-depression medication, she kept more to herself: "After I took the medicine, I almost refused the others... So, now at home, after eating, I just sit there and watch TV, like a fool. It was after taking the medication, that I stopped enjoying going out, things like that."

\section{Negative Attitudes about Social Services Related to Preference for Self-Reliance}

Five participants had not received any formal social services. In general, all participants, regardless of age or educational level, expressed feelings of self-sufficiency and negativity toward social services. They equated "needing help" with the stigma of "losing face." Their attitude was to not bother and burden others. They did not see the value of social services and were used to solving problems on their own. None expressed that physical distance and social distance, such as not speaking English, hindered them from accessing social services. Mrs. Wu explained:

"I am taking medicine and seem stable, so I don't need [social services]. In the past, I did not know about [social services]. Even if I wanted others to help me, I was embarrassed. I am a person who does not want to bother other people... My husband [also] does not want it. He wants to save face. [Our daughter is legally blind and my husband does not want to receive social services for her.]"

Only Mr. Zhen reported having received social services. He viewed the services, case management and in-home 
support services, as having made his life easier but he did not see them as having contributed to improving his mental health status and suicidal ideation. He argued that social services had not solved his problems, namely his depression, loneliness and anxiety. He felt much fear but did not know the source of his fear. He attempted suicide by overdosing in reaction to his loneliness and lack of sleep. He was still suicidal at the time of the study. He described his daily life as follows: "I don't want to read books or the newspaper. I don't want to watch TV. I just sit here alone and lonely... There is no joy in my life."

\section{Role of Family in Suicide Prevention}

Study participants reported that protection provided by one's family and close social network was important. All four women, representing diverse educational levels and age groups, named support from family and friends as critical protective factors. Only one man, Mr. Zhen did not cite family as a supportive factor in preventing suicidal behaviors. Although three of the study participants who were Christian, only Mrs. Wong identified her religion as a supportive factor alongside her family. For example, her father helped her out of her suicidal ideation and her friends assisted her financially:

"[My father] said that the whole family supported you. He said that my brothers supported me. The one in Canada came to take care of me when I had chemotherapy. [My father] said that so many people supported you, why did you want to go that direction [of suicide]... When I faced many difficulties, a family [who are my close friends] helped me with finances. They gave my daughter a job and stabilized our finances. The wife also helped me to get into [a clinical trial at the medical center]."

The four women also stated that their most important motivation for keeping themselves alive was their social responsibility toward their children.

\section{DISCUSSION}

Using a qualitative study method, the findings of our research provided numerous examples that demonstrate the influence of Chinese culture on immigrants' interpretations of suicidal distress, their need for help, and their conception of appropriate and useful services. Family-related difficulties figured prominently as reasons for suicide attempts; family members' denial of mental health issues contributed to the immigrants' lack of awareness about their mental health condition. Additionally, family denial of mental health issues and the weakening support of the family unit intertwined with poor linkage to the community exacerbated the distress. Other signs of lack of family support included attitudes about medications, family indifference, avoiding social stigma and not 'losing face', i.e., concerns about protecting one's family image. These concerns, while rarely stated directly, were the sources of negative attitudes toward mental health care and served as deterrents to using mental health facilities. Finally, those suffering from suicide ideation often had conceptions about acceptable means of help and linkage to care based on the attitudes of family, peers and the community.

Study participants' culturally based interpretations limited their recognition of the severity of their mental distress and contributed to delays in professional care until they actually made attempts to commit suicide. These family and cultural influences thus acted as barriers between the immigrant and available services. The experience of loss of face and stigma of mental illness kept participants from accessing these forms of help before and after their suicide attempts. If a person, even after attempting suicide, will not access such services, their risk of suicide may not be mitigated. Such resistant and high risk attitudes and help seeking behaviors point to the importance of creative educational or preventative efforts to change cultural attitudes about services and gain acceptance in the community for these services.

Some of our interviewees revealed how the stigma of mental illness, which brings shame to the family, might have exacerbated the attempt to end one's life. This shame, coupled with acculturative stress and family conflict, are recognized correlates of suicide among Chinese Americans. Our interviews highlighted the experience of isolation as an important factor leading to study participants' attempted suicide, and also their own and their families' lack of recognition or denial that they were experiencing a mental health crisis. Perhaps the most important findings from this small exploratory study derive from how the interviews revealed the key mechanisms behind the experience of isolation and acculturation stress. These are the progressive loss of family support from one's home country, poor integration due to language, and disharmony among family members due to acculturative differences.

When we consider our study findings in the context of the three dynamic models of help seeking, we observed that these models may have applicability and utility for future research in the Chinese American immigrant population. The cycle of avoidance model highlights ambivalence and difficulties experienced which prevent people from receiving adequate care [30]. While our study participants did not particularly express ambivalence, they did describe their difficulties resulting from the strong influence of their families who sometimes shut down all pathways by not recognizing their distress or denigrating mental health services. Family members also misconceived the severity of suicidal behaviors, and the social meaning attached to help seeking was generally one of social stigma. The NetworkEpisode Model highlights the impact of the social support system's attitudes on individual actions [31]. Applying this model, the process of social influence by the immigrants' families was prominent and strong, and their negative attitudes toward the use of mental health services contributed to denying the need for care. The concept of help-seeking pathways [34] is helpful for understanding evolving cultural interpretations of the distress related to both family and community.

Study participants appeared to see the value of an efficient ordering of services as helpful. Interviews reveal that study participants did not reach professional services for a very long time and as a suggestion, they pointed to the need of outreach and changes in the socio-cultural context so 
that others who experience their situation would reach professional care sooner, cutting the duration from when the immigrant initiates help seeking interpersonally and when they reach professional care. Considering weakened support from family members who remained in countries of origin as inevitable, findings suggest that it is necessary to devise improved means of linkage with the immigrant's new and old social networks. Alternative social networks could be built from the beginning of the immigrant's stay in the U.S. or interventions could be developed to build individual resilience, including through improved use of information and communications technology.

\section{CONCLUSION}

One of the implications of the study is that the mental health needs of this population are poorly accommodated by the existing mental health system. Study participants expressed negative attitudes toward existing services, including counseling, mental health treatment and social services. To them, service delivery methods demonstrated a poor fit between these services and the immigrants' perception of their needs. If current services remain nonaccessible for cultural reasons even after a suicide attempt, it is important to understand at-risk immigrants' conception of "acceptable and needed forms of help". Our interviewees emphasized the critical role of outreach, especially community-based and peer outreach. Given Chinese American immigrants' experiences of fear and selfdistancing, forms of effective outreach and linkage to care forms of effective outreach and linkage to care that are physically, socially and culturally acceptable become the most urgent objective in designing social service for this community.

The strength of our study is that it provides the 'lived experience' of a group of Chinese immigrants in California on this important topic. Their words provide a real-life example of how concepts like family in Chinese culture and acculturation stress play out in attitudes and behaviors regarding help seeking. However, this study is limited by its small sample size, and the interview with respondents was within one week of their suicide attempts. With regard to the small sample, it was not possible to achieve saturation of the data and infer higher risks or differences between subgroups. A larger sample representing more diverse backgrounds would enrich the data and permit the observation of expression pattern of distress and help seeking behaviors. With regard to interviewing respondents very shortly after their suicide attempts, the views expressed may be tinted due their unstable emotional states.

This study provides a good picture of the needs of Chinese immigrants living in the San Francisco Bay Area. Drawing implications from this study to immigrants in California coming from other countries and other socioeconomic backgrounds may be misleading since Asian culture is very diverse. However, this study does bring needed attention to important issues in the Chinese American immigrant community. It is a start - a badly needed reminder of the need to move beyond the Eurocentric perspective to understand the hurts and pains and solutions for minority populations. One of the follow-up projects that grew out of this study was the development of culture-specific, Chinese language educational videos and symptom checklists for depression that address family- and acculturation-related stressors created using a community-based participatory research approach [36]. These materials are specifically intended to support outreach efforts in the community context. With the continued influx of Chinese immigrants to the U.S., more culture-specific outreach programs targeting this population are needed to reach those who are experiencing serious bio-psycho-social risk factors related to suicide and poor access to care detected in this study.

\section{CONFLICT OF INTEREST}

The authors confirm that this article content has no conflict of interest.

\section{ACKNOWLEDGEMENTS}

Declared none.

\section{REFERENCES}

[1] Cheng JKY, Fancher TL, Ratanasen M, et al. Lifetime suicidal ideation and suicide attempts in Asian Americans. Asian Am J Psychol 2010; 1(1): 18-30.

[2] Xu J, Kochanek KD, Murphy SL, Tejada-Vera B. Deaths: final data for 2007. Natl Vital Stat Rep 2010; 58: 10.

[3] Kessler RC, Borges G, Walters EE. Prevalence of and risk factors for lifetime suicide attempts in the national comorbidity survey. Arch Gen Psychiatry 1999; 56 (7): 617-26.

[4] Centers for Disease Control and Prevention, National Center for Injury Prevention and Control. Web-based Injury Statistics Query and Reporting System (WISQARS) [internet]. Washington, D.C.: Author; 2010 [cited 30 Sep 2014]. Available from: www.cdc.gov/ injury/wisqars/index.html

[5] Heron M. Deaths: leading causes for 2007. Natl Vital Stat Rep 2011; 59(8): 1-95.

[6] Bartels SJ, Coakley E, Oxman TE, et al. Suicide and death ideation in older primary care patients with depression, anxiety, and at-risk alcohol abuse. Am J Geriatr Psychiatry 2002; 10(4): 417-27.

[7] Abe-Kim J, Takeuchi DT, Hong S, et al. Use of mental health related services among immigrants and U.S. born Asian Americans: results form the National Latino and Asian American Study. Am J Public Health 2007; 97(1): 91-8.

[8] Zhang AY, Snowden LR, Sue S. Differences between Asian and White Americans' help-seeking and utilization patterns in the Los Angeles area. J Community Psychol 1998; 26(4): 317-326.

[9] Alegria M, Chatterji P, Wells $\mathrm{K}$, et al. Disparity in depression treatment among racial and ethnic minority populations in the U.S. Psychiatry Serv 2008; 59(11): 1264-72.

[10] Chu JP, Hsieh K-Y, Tokars DA. Help-seeking tendencies in Asian Americans with suicidal ideation and attempts. Asian Am J Psychol 2011; 2(1): 25-38.

[11] Chung I. Changes in the sociocultural reality of Chinese immigrants: challenges and opportunities in help-seeking behavior. Int J Soc Psychiatry 2010; 56(4): 436-47.

[12] Chung I. Sociocultural study of immigrant suicide-attempters: an ecological perspective. J Soc Work 2011; 12(6): 614-29.

[13] Morrison LL, Downey DL. Racial differences in self-disclosure of suicidal ideation and reasons for living: implications for training. Cultur Divers Ethnic Minor Psychol 2000; 6(4): 374-86.

[14] Lee SM. Asian Americans struggle with suicide. SFGate [Internet]. 2013 Jan 24 [cited 2014 Sep 30]. Available from: http://www.sfg ate.com/health/article/Asian-Americans-struggle-with-suicide4214483.php\#photo-4066399

[15] Wong YJ, Uhm SY, Li P. Asian Americans' family cohesion and suicide ideation: moderating and mediating effects. Am J Orthopsychiatry 2012; 82(3): 309-18.

[16] Chen AW, Kazanjian A, Wong H. Determinants of mental health consultations among recent Chinese immigrants in British Columbia, Canada: implications for mental health risk and access to services. J Immigr Minor Health 2008; 10(6): 529-40. 
[17] Leong FTL, Wagner NS, Tata SP. Handbook of multicultural counseling: racial and ethnic variations in help-seeking attitudes. Thousand Oaks, CA: Sage 1995.

[18] Cheng JKY, Fancher TL, Ratanasen M, et al. Lifetime suicidal ideation and suicide attempts in Asian Americans. Asian Am J Psychol 2010; 1(1): 18-30.

[19] Shiang J. Review of suicidology: considering cultural beliefs and behaviors in the study of suicide. New York: Guilford Press 2000.

[20] Kung W. Cultural and practical barriers to seeking mental health treatment for Chinese Americans. J Commun Psychol 2004; 32(1): 24-37.

[21] Hwang W, Myers H, Abe-Kim J, Ting J. A conceptual paradigm for understanding culture's impact on mental health: the cultural influences on mental health (CIMH) model. Clin Psychol Rev 2008; 28(2): 211-27.

[22] Kim BSK. Handbook of Asian American psychology: acculturation and enculturation. Thousand Oaks, CA: Sage 2007.

[23] Koss-Chioino JD. Psychological intervention and cultural diversity: traditional and folk approaches among ethnic minorities. Needham Heights, MA: Allyn \& Bacon 2000.

[24] Hong GK, Lee BS, Lorenzo MK. Somatization in Chinese American clients: implications for psychotherapeutic services. J Contemp Psychother 1995; 25(2): 105-18.

[25] Ying YW. Explanatory models of major depression and implications for help-seeking among immigrant Chinese-American women. Cult Med Psychiatry 1990; 14(3): 393-408.

[26] Akutsu PD, Chu JP. Clinical problems that initiate professional help-seeking behaviors from Asian Americans. Prof Psychol 2006; 37(4): 407-15.

[27] Lin KM, Cheung F. Mental health issues for Asian Americans. Psychiatry Serv 1999; 50(6): 774-80.
[28] Liu Y. Rethinking emotions in Confucian political philosophy. J East West Thought 2014; 1(4): 43-57.

[29] Hsiao FH, Klimidis S, Minas H, Tan ES. Cultural attribution of mental health suffering in Chinese societies: the views of Chinese patients with mental illness and their caregivers. J Clin Nurs 2006; 15(8): 998-1006.

[30] Pescosolido BA, Boyer CA. How do people come to use mental health services? Current knowledge and changing perspectives. In: Horwitz AV, Scheid, Eds. Handbook for the study of mental health: social contexts, theories \& systems. New York: Cambridge University Press 1999; pp. 392-411.

[31] Biddle L, Donovan J, Sharp D, Gunnell D. Explaining non-helpseeking amongst young adults with mental distress: a dynamic interpretative model of illness behavior. Sociol Health Ill 2007; 29(7): 983-1002.

[32] Kadushin, C. The friends and supporters of psychotherapy: on social circles in urban life. Am Soc Rev 1966; 31:786-802.

[33] Pescosolido BA, Wright ER, Alegria M, Vera M. Social networks and patterns of use among the poor with mental health problems in Puerto Rico. Med Care 1998; 36(7): 1057-72.

[34] Rogler LH, Cortes DE. Help-seeking pathways: a unifying concept in mental health care. Am J Psychiatry 1993; 150(4): 554-61.

[35] Strauss A, Corbin JM. Basics of qualitative research: grounded theory procedures and techniques. Newbury Park, CA: Sage 1990.

[36] Wong R, Lim-Yee N, Wu R, Guo C. Addressing mental health disparities through community-based participatory research: development of culturally sensitive assessment and educational materials for depression Chinese immigrants in the United States. Paper presented at: Global Social Sciences Conference, Department of Social Work, Hong Kong Baptist University, April 10-12; Hong Kong, People's Republic of China 2014.

Received: October 23, 2014

(C) Wong et al.; Licensee Bentham Open.

This is an open access article licensed under the terms of the Creative Commons Attribution Non-Commercial License (http://creativecommons.org/licenses/by-nc/3.0/) which permits unrestricted, non-commercial use, distribution and reproduction in any medium, provided the work is properly cited. 